OPEN ACCESS

Edited by:

Yan-yan Yan

Shanxi Datong University, China

Reviewed by:

Zui Pan,

University of Texas at Arlington,

United States

Hui Yang,

Second Affiliated Hospital of Guangzhou Medical University, China

${ }^{*}$ Correspondence:

Zhi Shi

tshizhi@jnu.edu.cn

†These authors have contributed equally to this work

Specialty section:

This article was submitted to Cancer Molecular Targets and

Therapeutics,

a section of the journal

Frontiers in Oncology

Received: 25 December 2018

Accepted: 29 January 2019

Published: 27 February 2019

Citation:

Wang K, Xing Z-H, Jiang Q-W, Yang Y, Huang J-R, Yuan M-L, Wei M-N, Li Y,

Wang S-T, Liu K and Shi Z (2019)

Targeting UPAR by CRISPR/Cas 9

System Attenuates Cancer

Malignancy and Multidrug Resistance.

Front. Oncol. 9:80

doi: 10.3389/fonc.2019.00080

\section{Targeting uPAR by CRISPR/Cas9 System Attenuates Cancer Malignancy and Multidrug Resistance}

\author{
Kun Wang ${ }^{\dagger}$, Zi-Hao Xing ${ }^{\dagger}$, Qi-Wei Jiang, Yang Yang, Jia-Rong Huang, Meng-Ling Yuan, \\ Meng-Ning Wei, Yao Li, Sheng-Te Wang, Kun Liu and Zhi Shi*
}

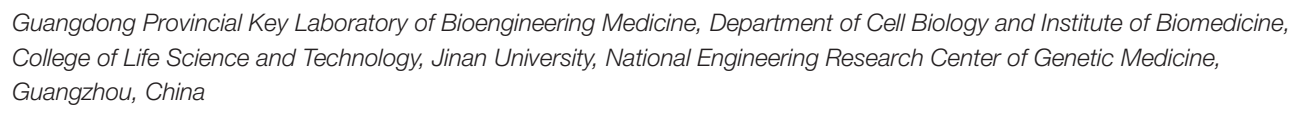

Urokinase plasminogen activator receptor (UPAR), a member of the lymphocyte antigen 6 protein superfamily, is overexpressed in different types of cancers and plays an important role in tumorigenesis and development. In this study, we successfully targeted uPAR by CRISPR/Cas9 system in two human cancer cell lines with two individual sgRNAs. Knockout of UPAR inhibited cell proliferation, migration and invasion. Furthermore, knockout of uPAR decreases resistance to 5-FU, cisplatin, docetaxel, and doxorubicin in these cells. Although there are several limitations in the application of CRISPR/Cas9 system for cancer patients, our study offers valuable evidences for the role of UPAR in cancer malignancy and drug resistance.

\section{Keywords: cancer, uPAR, CRISPR/Cas9, malignancy, drug resistance}

\section{INTRODUCTION}

Urokinase plasminogen activator (UPA) receptor (UPAR), also known as CD87 and encoded by PLAUR gene, is a member of the lymphocyte antigen 6 protein superfamily (1). uPAR is a glycoprotein consisting of 313 amino acid residues with only the extracellular domain, no transmembrane and intracellular structures, and is attached to the cell membrane via glycosylphosphatidylinositol anchors (1). uPAR binds to and activates uPA to cleaving plasminogen to plasmin, thus triggering the remodeling of extracellular matrix and playing a key role in cell adhesion, migration, proliferation, and survival (2). Besides uPA, uPAR can interact with other proteins, including vitronectin, integrins, and EGFR, etc to regulate multiple signal pathways (2). Compared to normal tissues, uPAR is highly expressed in many human cancers including lung, breast, gastric, colorectal, pancreatic, bladder, and prostate cancers, etc (3). The expression of uPAR in these cancers promotes the proliferation, metastasis, and invasion of cancer cells (3). Therefore, uPAR may be an important biomarker and target for cancers. Indeed, many inhibitors of uPAR have been developed. The inhibitors blocks the interaction of uPAR with uPA, including: small molecules UK1 (4), WX-UK1 (5), WX-671 (6), etc; peptides Mupain-1 (7), AE105 (8), ATF (9), etc; and monoclonal antibody ATN-291 (10). In addition, there are inhibitors that inhibit the interaction of uPAR with integrins, including: peptides P25 (11), a325 (12), H245A (13), etc; and monoclonal antibody ATN-658 (14). However, the poor affinity and bioavailability limit the application of these inhibitors in clinic. Consequently, it is necessary to develop new approaches to target uPAR for treatment cancer and other diseases. 
The RNA-guided clustered regularly interspaced short palindromic (CRISPR) in combination with a CRISPRassociated nuclease 9 (Cas9) nuclease system is a novel gene editing technology by delivering the Cas9 complexed with a synthetic guide RNA (gRNA) into a cell to cut the desired genome location, allowing existing genes to be removed and/or new ones added (15). Due to the advantages of faster, cheaper, more accurate, and efficient, CRISPR/Cas9 system has been widely used as a basic biology research tool, development of biotechnology products and potentially to treat diseases (16). In this study, we used CRISPR/Cas9 system targeting uPAR to verify the role of UPAR in cancers.

\section{MATERIALS AND METHODS Cells and Reagents}

The two multidrug resistant cancer cell lines HCT8/T and $\mathrm{KB}_{\mathrm{V} 200}$ were cultured in Dulbecco's modified Eagle's medium (DMEM) with $10 \% \mathrm{FBS}$, penicillin $(100 \mathrm{U} / \mathrm{ml})$ and streptomycin $(100 \mathrm{ng} / \mathrm{ml})$ at $37^{\circ} \mathrm{C}$ in a humidified atmosphere of $5 \%$ $\mathrm{CO}_{2}$. Restriction endonuclease BsmBI was from New England Biolabs. Polyetherimide (PEI) was from Ploysciences. Cisplatin was from Shandong Qilu Pharmaceutical. 5-FU, docetaxel, and doxorubicin were from LC Laboratories. Puromycin was from Selleck Chemicals. Methylthiazolyldiphenyl-tetrazolium bromide (MTT) was from ApexBio Technology. Anti-uPAR (D121140) antibody was from Shanghai sangon biotech. AntiVinculin antibody (BM1611) was from Wuhan Boster Biotech.

\section{Vector Generation, Lentivirus Production, and Transduction}

LentiCRISPRv2 vector (from Addgene \#52961) was digested with BsmBI and ligated with annealed oligonucleotides (uPAR-sg1F: 5'-CACCGGACCAACGGGGATTGCCGTG-3', uPAR-sg1R: 5'-AA-ACCACGGCAATCCCCGTTGGTCC-3'; uPAR-sg2-F: 5'-CACCGGGACCACGATCGTGCGCTTG-3', uPAR-sg2-R: 5' AAACCAAGCGCACGATCGTGGTCCC-3'). HEK293T were transfected using PEI at $70 \%$ confluency with recombinant vectors and packaging vectors $\mathrm{pMD} 2 \mathrm{G}$ and psPX2. Viral supernatant was harvested $96 \mathrm{~h}$ after transfection and stored at $-80^{\circ} \mathrm{C}$. HCT-8/T and $\mathrm{KB}_{\mathrm{V} 200}$ cells were transducted with viral supernatant containing $10 \mu \mathrm{g} / \mathrm{ml}$ polybrene, and were selected with 100 and $10 \mu \mathrm{g} / \mathrm{ml}$ puromycin respectively to establish the stable cell lines.

\section{Genomic PCR and Sequencing Analysis}

The genomic DNA of cells was extracted with the QuickExtract DNA extraction kit following the manufacturer's protocol and amplified with a pair of primers (Detection 1-F: 5'-GACAACGGACAGACTGGAA-3', Detection 1-R: 5' CCGAATCGCTCTAAGTGG-3') designed for the target region of interest using a Pfu DNA polymerase. Followed by agarose gel electrophoresis and ethidium bromide staining, the purified PCR products were sequencing with an ABI 3131xl Genetic analyzer.

\section{Western Blot Analysis}

Cells were harvested and lysed in RIPA buffer (1\% NP$40,0.5 \%$ sodium deoxycholate, $0.1 \%$ SDS, $10 \mathrm{ng} / \mathrm{ml}$ PMSF, $0.03 \%$ aprotinin, $1 \mu \mathrm{M}$ sodium orthovanadate) at $4{ }^{\circ} \mathrm{C}$ for $30 \mathrm{~min}$. Lysates were centrifuged for $10 \mathrm{~min}$ at $14,000 \times \mathrm{g}$ and supernants were stored at $-80^{\circ} \mathrm{C}$ as whole cell extracts. Protein concentration was quantified using with Bradford assay. Proteins were separated on 10\% SDS-PAGE gels and transferred to polyvinylidene difluoride membranes. Membranes were blocked with 5\% BSA and incubated with the indicated primary antibodies. Corresponding horseradish peroxidase-conjugated secondary antibodies were used against each primary antibody. Proteins were detected using the chemiluminescent detection reagents and films.

\section{Cell Morphology Assay}

Cells were seeded on glass cover slips for $24 \mathrm{~h}$ and then fixed in $4 \%$ paraformaldehyde for $20 \mathrm{~min}$ and permeabilized with $0.1 \%$ Triton X-100 for $15 \mathrm{~min}$ at room temperature. The coverslips were incubated in the dark with $100 \mathrm{nM}$ rhodamine-phalloidin at room temperature for $30 \mathrm{~min}$. Nuclei were counterstained with $100 \mathrm{nM}$ DAPI. The coverslips were rinsed in PBS and inverted on a drop of anti-fade mounting media on a glass slide. Then, these slides were sealed with neutral balsam and viewed under the confocal microscope.

\section{Cell Viability Assay}

Cells were seeded into a 96-well plate at a density of 5,000 cells/well and treated with various concentrations of agents for $72 \mathrm{~h}$. Then $10 \mu \mathrm{l} \mathrm{MTT}$ was added to each well at a final concentration of $0.5 \mathrm{mg} / \mathrm{ml}$. After incubation for $4 \mathrm{~h}$, formazan crystals were dissolved in $50 \mu \mathrm{l}$ of DMSO, and absorbance at $570 \mathrm{~nm}$ was measured by plate reader. The concentrations required to inhibit growth by $50 \%\left(\mathrm{IC}_{50}\right)$ were calculated from survival curves as previously described (17).

\section{Sphere Formation Assay}

Cells were trypsinized, suspended in medium containing $0.3 \%$ agar and $10 \%$ FBS and seeded at a density of $5 \times 10^{2}$ cells/well in a 12 -well plate. The agar-cell mixture was plated onto a bottom layer with $0.5 \%$ agar. Then treated cells were incubated in a humidified incubator and fresh medium was added every 3 days. Two weeks later, colonies were analyzed microscopically.

\section{Cell Migration Assay}

Cells were seeded into a 6-well plate, and reached $80-90 \%$ confluence, the cell monolayer was wounded using a sterilized $10 \mu \mathrm{l}$ pipette tip and washed with PBS two times. Cells were allowed to migrate for 12,24 , and $36 \mathrm{~h}$ in serum-free medium, and the wounds were observed and captured. The gap lengths were measured from the photomicrographs.

\section{Cell Invasion Assay}

Cell invasion assays were performed with a modified Boyden chamber (Corning) containing matrigel-coated polycarbonate membrane filter $(6.5 \mathrm{~mm}$ diameter, $8 \mu \mathrm{m}$ pore size). Cells were plated in the upper chamber and the lower chamber contained medium with $10 \%$ FBS, and incubated for $24 \mathrm{~h}$ 
at $37^{\circ} \mathrm{C}$ in $5 \% \mathrm{CO}_{2}$. Non-migrated cells were scraped from the upper surface of the membrane, and migrated cells remaining on the bottom surface were photographed and counted.

\section{Statistical Analysis}

The experimental data of this paper are the results of three independent repetitions. The data obtained is presented in the form of an average and a standard deviation. Statistical analysis

A

lentiCRISPRv2 :

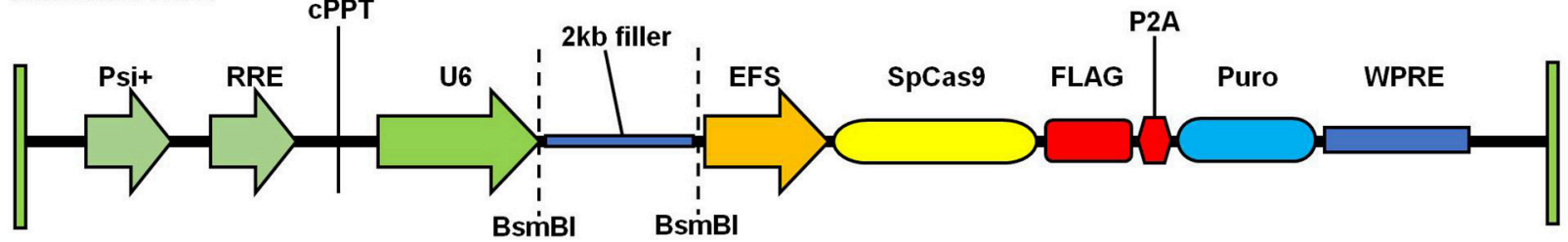

B

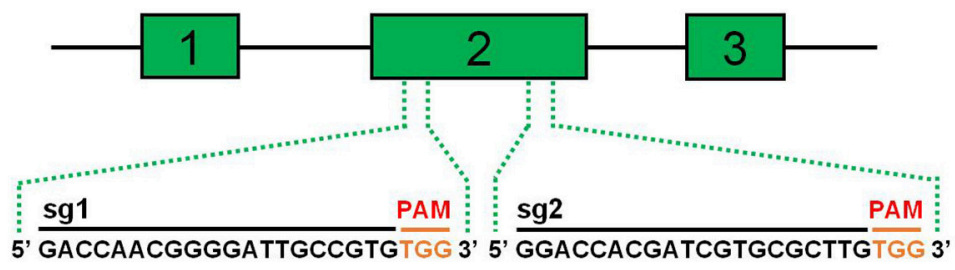

C

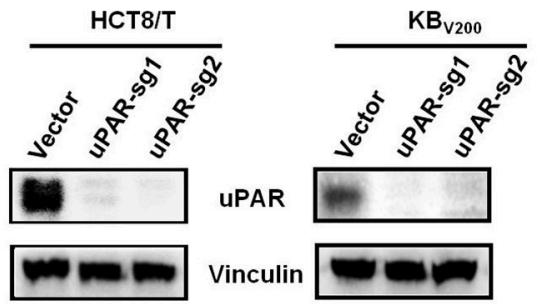

D
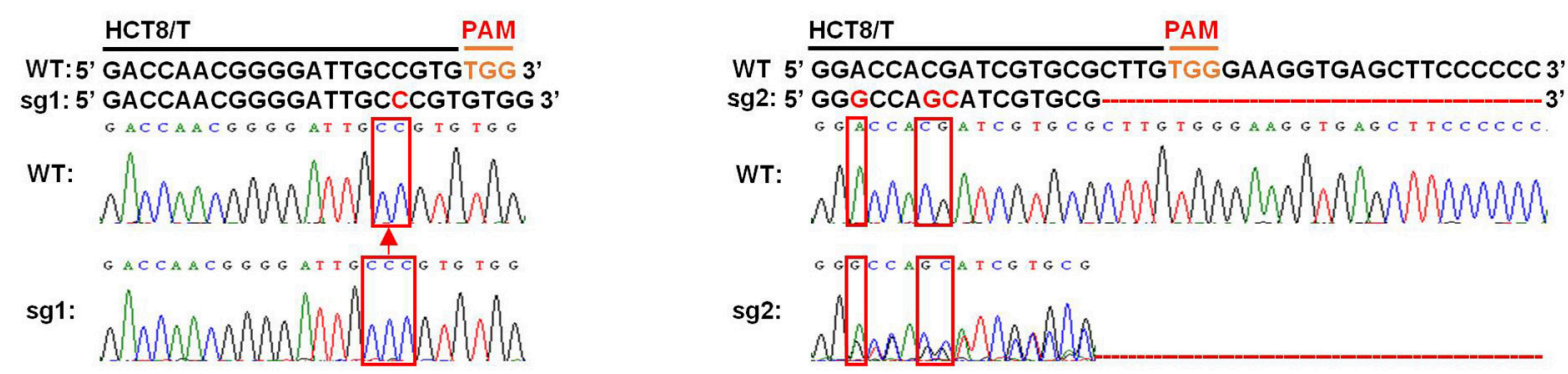

E

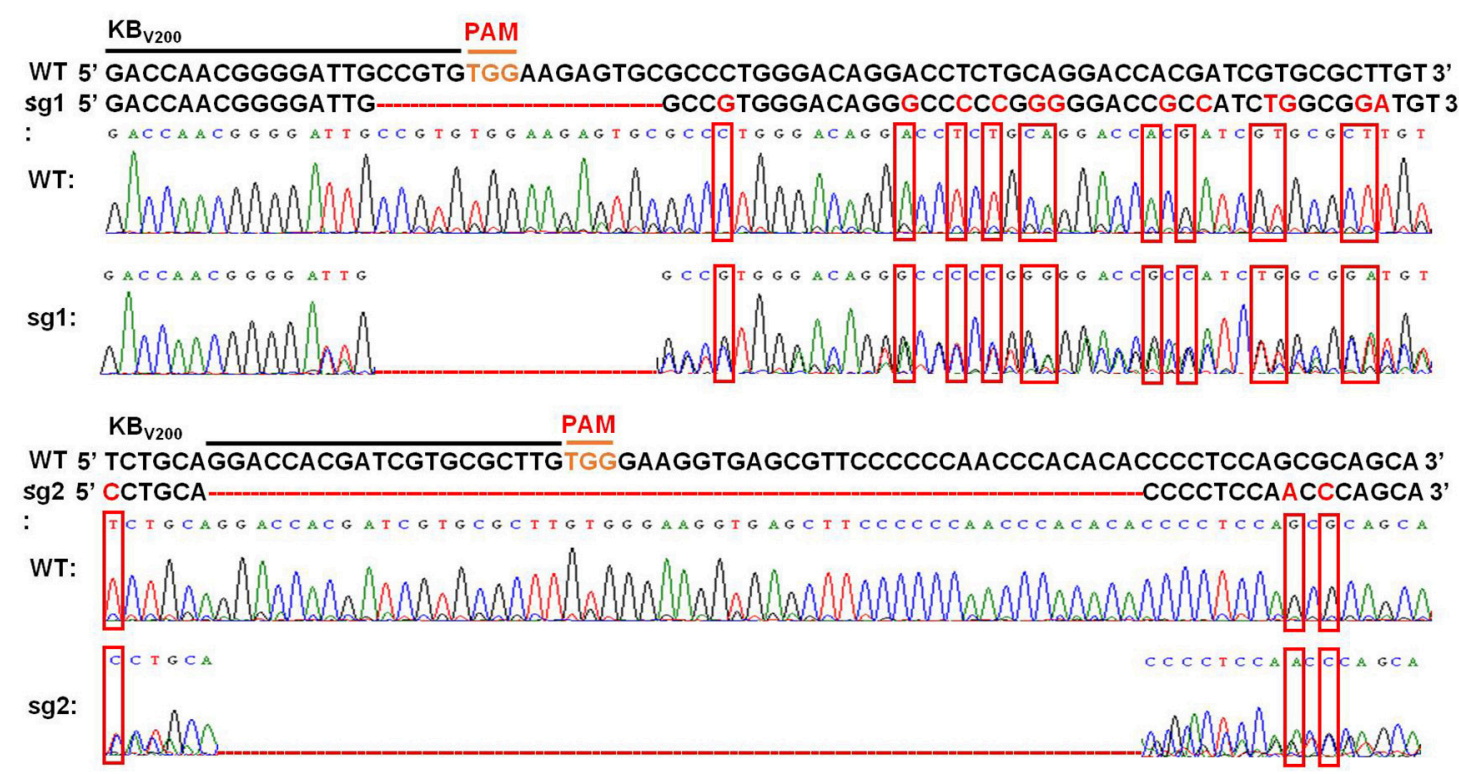

FIGURE 1 | Knockout of uPAR by CRISPR/Cas9 system. (A) The map of lentiCRISPRv2 vector. (B) The locations and sequences of two sgRNAs of uPAR. (C) The protein expression levels of UPAR were examined by Western blot, and vinculin was used as loading control. The genomic DNA of cells was amplified and sequenced by the designed primers. The sequencing comparison and original data of HCT8/T (D) and KBV200 (E) cells are shown. 
of data differences using $t$-test method. A $P$-value of $<0.05$ was set as the criterion for statistical significance.

\section{RESULTS}

\section{Knockout of uPAR by CRISPR/Cas9 System}

To target uPAR with CRISPR/Cas9 system, we firstly used lentiCRISPRv2 vector which expresses both hSpCas9 and the chimeric guide RNA (Figure 1A) linked respectively, with two targeting sequences from exon 2 of human uPAR gene (PLAUR) end with a 5'NGG3' PAM (protospacer adjacent motif) sequence (Figure 1B). Then, the two successfully generated vectors expressed sgRNA1 (sg1) or sgRNA2 (sg2) to target UPAR were identified by sequencing. To establish cell lines stably expressed sgRNA to target uPAR, HCT8/T, and $\mathrm{KB}_{\mathrm{V} 200}$ cells were selected with puromycin after transduction with LentiCRISPRv2 viral supernatant. As shown in Figure 1C, the protein levels of $\mathrm{UPAR}$ were undetectable by western blot in both $\mathrm{HCT} 8 / \mathrm{T}$ and $\mathrm{KB}_{\mathrm{V} 200}$ cells stably expressed either sg1 or sg2. To further identify the genomic change of targeting uPAR by CRISPR/Cas9 system, the genomic DNA of cells was extracted and amplified using the designed primers by PCR reaction. The sequencing results of PCR productions showed that 1 base was inserted into the target position of HCT8/T uPAR-sg1 cells and 3 base mismatches and a large deletion in the target position of HCT8/T uPAR-sg2 cells (Figure 1D). There were 16 base deletions and 12 base mismatches in the target position of $\mathrm{KB}_{\mathrm{V} 200}$ uPAR-sg1 cells and 51 base deletions and 3 base mismatches in the target position of $\mathrm{KB}_{\mathrm{V} 200}$ uPAR-sg2 cells (Figure 1E). These data suggest that cells with stable knockout of uPAR by CRISPR/Cas9 system were successfully established.

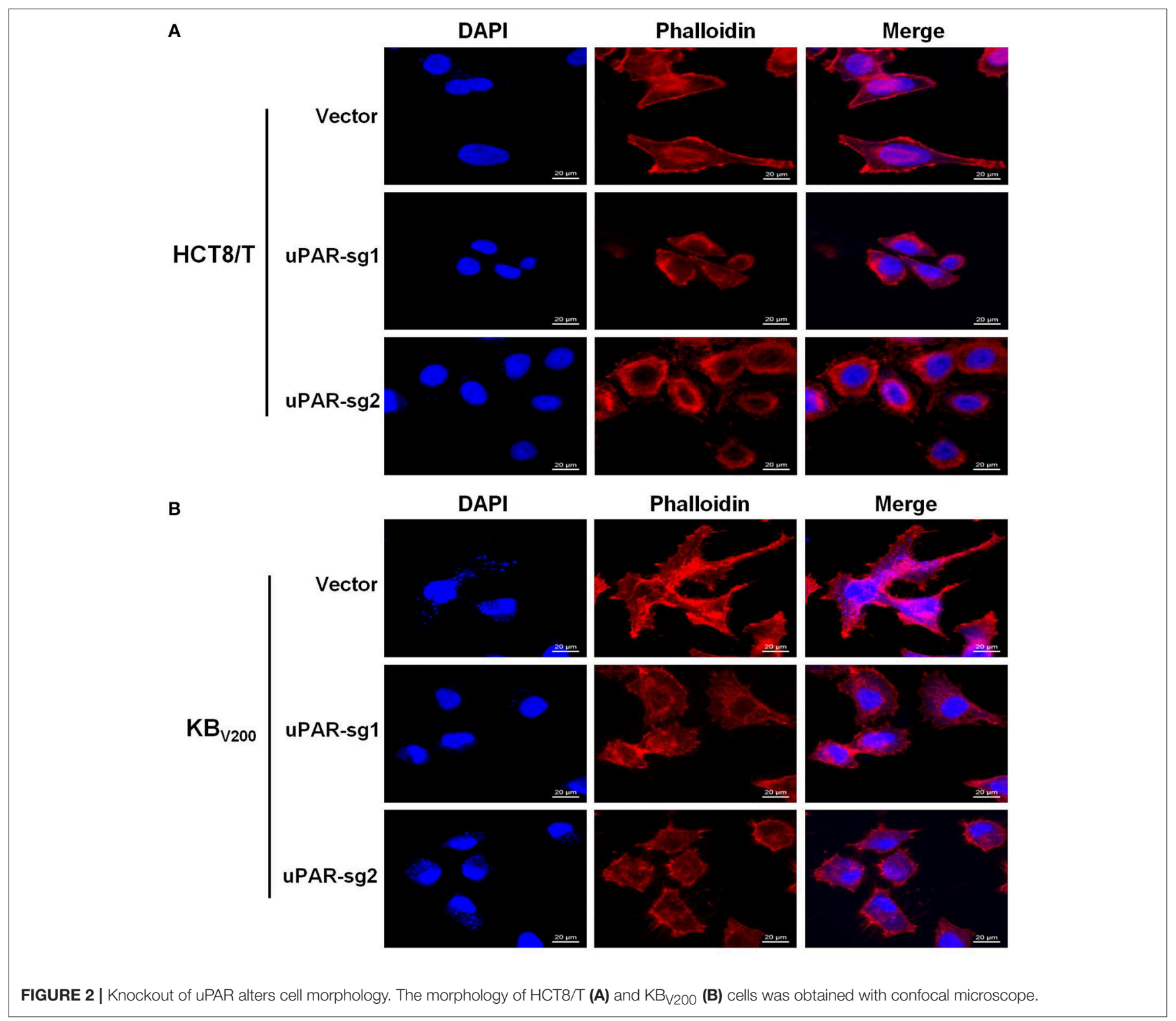




\section{Knockout of uPAR Alters Cell Morphology}

To explore the effect of knockout of uPAR on cell morphology, we stained cells with Rhodamine-labeled phalloidin and DAPI. The results showed that $\mathrm{HCT} 8 / \mathrm{T}$ and $\mathrm{KB}_{\mathrm{V} 200}$ cells with uPAR knockout underwent morphologic changes from spindle-shaped phenotype to round phenotype (Figures $2 \mathrm{~A}, \mathbf{B}$ ), indicating that knockout of uPAR alters cell morphology.

\section{Knockout of uPAR Attenuates Cell Proliferation}

To investigate the effect of knockout of uPAR on cell proliferation, we detected cell proliferation by MTT and sphere formation assays. As shown in Figure 3A, knockout of uPAR inhibited the growth of HCT8/T and $\mathrm{KB}_{\mathrm{V} 200}$ cells. Further sphere formation assay showed that knockout of uPAR reduced the sphere number and size of HCT8/T and $\mathrm{KB}_{\mathrm{V} 200}$ cells (Figures 3B-E). These results suggest that knockout of uPAR attenuates cell proliferation.

\section{Knockout of uPAR Inhibits Cell Migration}

To examine the effect of knockout of uPAR by CRISPR/Cas9 on cell migration, wound healing assay was used to detect cell migration. The results showed that cell migration was reduced in $\mathrm{HCT} 8 / \mathrm{T}$ and $\mathrm{KB}_{\mathrm{V} 200}$ cells with uPAR knockout (Figure 4), indicating that knockout of uPAR inhibits cell migration.
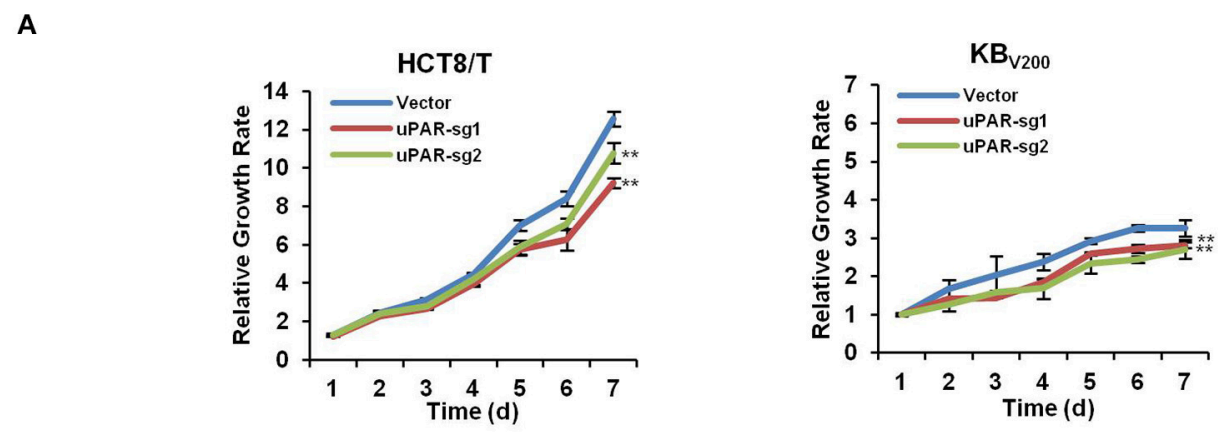

B

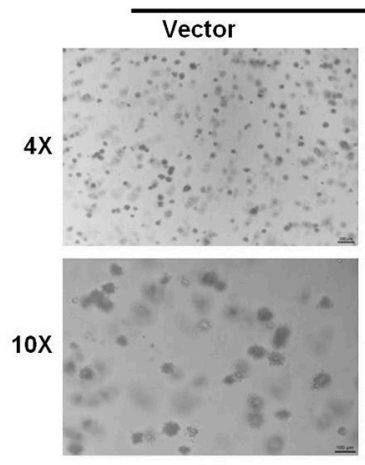

D

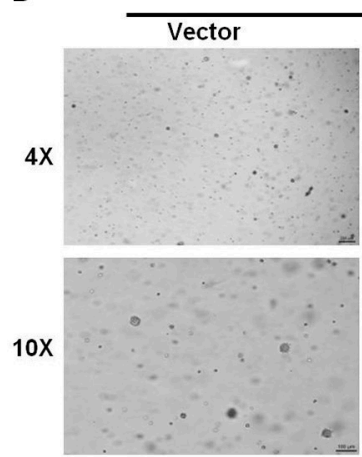

HCT8/T

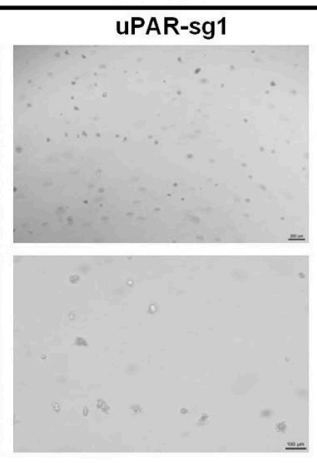

$\mathrm{KB}_{\mathrm{V} 200}$ uPAR-sg1
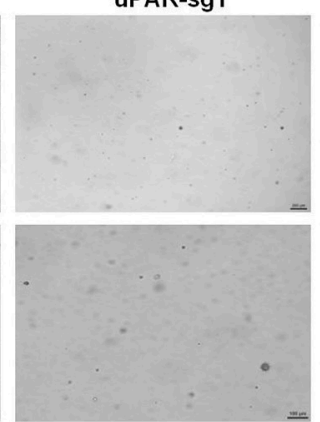

uPAR-sg2

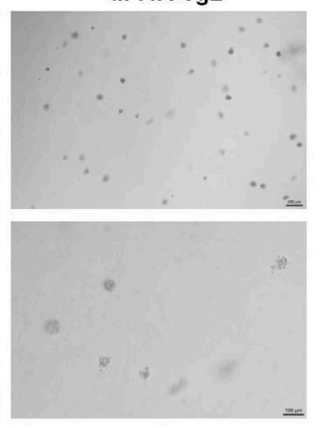

uPAR-sg2

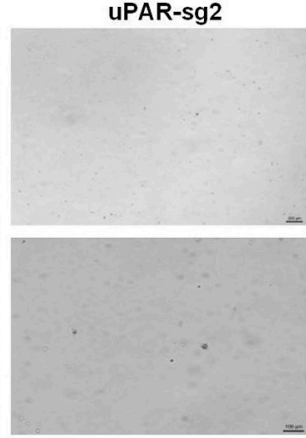

C

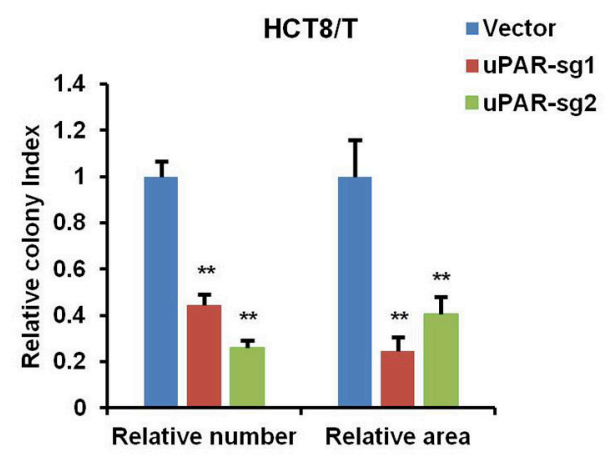

E

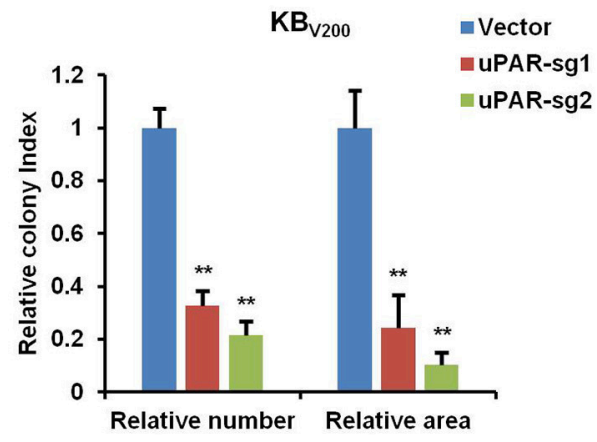

FIGURE 3 | Knockout of UPAR attenuates cell proliferation. (A) Cell proliferation was evaluated by MTT assay. Representative spheres images and quantification of $\mathrm{HCT8/T} \mathbf{( B , C )}$ and KBV200 (D,E) cells were determined by sphere formation assay. ${ }^{* *} P<0.01$ vs. corresponding control. 


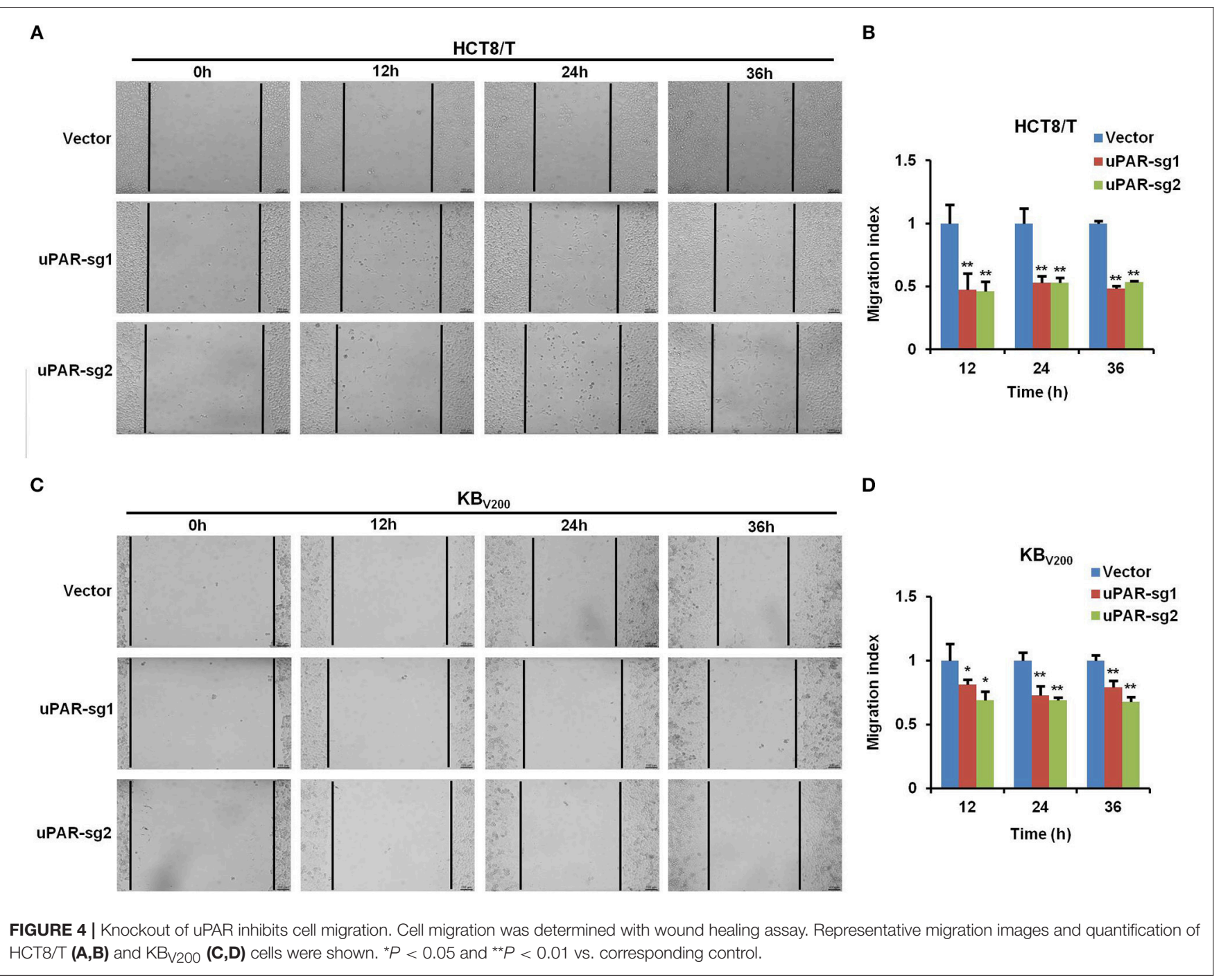

\section{Knockout of uPAR Inhibits Cell Invasion}

To further evaluate the effect of knockout of uPAR by CRISPR/Cas9 on cell invasion, transwell assay was used to detect cell invasion. As shown in Figure 5, cell invasion was reduced in HCT8/T and $\mathrm{KB}_{\mathrm{V} 200}$ cells with uPAR knockout, suggesting that knockout of UPAR inhibits cell invasion.

\section{Knockout of uPAR Decreases Multidrug Resistance}

To study the effect of knockout of uPAR by CRISPR/Cas9 on multidrug resistance, four chemotherapeutical drugs 5-FU, cisplatin, docetaxel, and doxorubicin were used to treat cells, and cell survival was detected by MTT assays. As shown in Figure 6, the cell survival curves shifted to downward, and $\mathrm{IC}_{50}$ values of these four drugs were reduced in $\mathrm{HCT} 8 / \mathrm{T}$ and $\mathrm{KB}_{\mathrm{V} 200}$ cells with uPAR knockout. These data indicate that knockout of uPAR suppresses multidrug resistance.

\section{DISCUSSION}

Recently, it has been demonstrated that knockout of uPAR using CRISPR/Cas9 system in mouse neuroblastoma Neuro 2A cells inhibit cell proliferation, reduce the number of Ki-67 positive cells, and down-regulate the mRNA expression level of $\mathrm{TrkC}$ receptor (18). In the current study, we successfully targeted uPAR in two cancer cell lines by CRISPR/Cas9 system with two individual sgRNAs. Knockout of uPAR suppresses cell proliferation, migration and invasion. Moreover, knockout of UPAR decreases resistance to 5-FU, cisplatin, docetaxel, and doxorubicin in these cells. Previous studies have shown that high expression of uPAR leads to small cell lung cancer, head and neck squamous cell carcinoma, and malignant pleural mesothelioma resistant to chemotherapy (19-21). uPAR promotes the resistance to tamoxifen in breast cancer by activated ERK1/2 activity (22), and confers the resistance to gefitinib in non-small-cell lung cancer through activated EGFR/pAKT/survivin signal pathway (23). Therefore, uPAR plays important roles not only in cancer malignancy but also in drug resistance. 
A

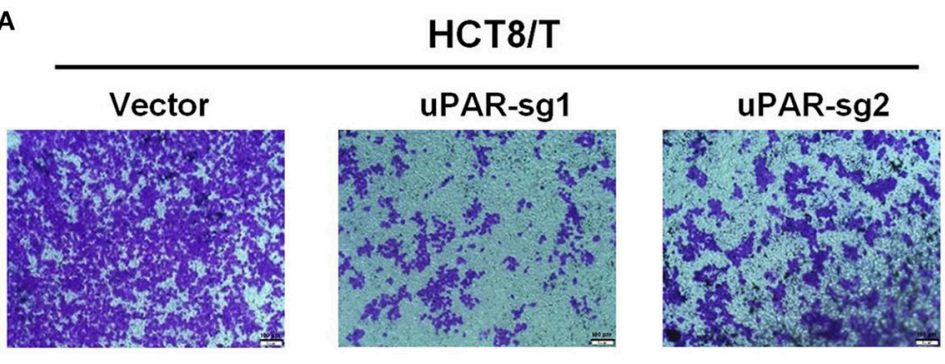

C

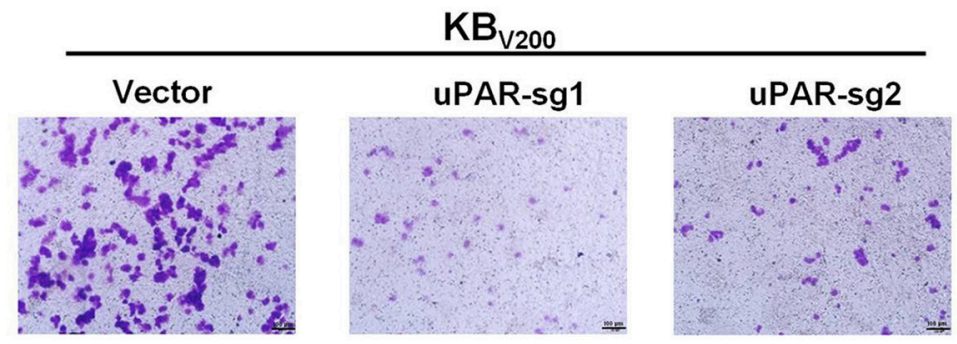

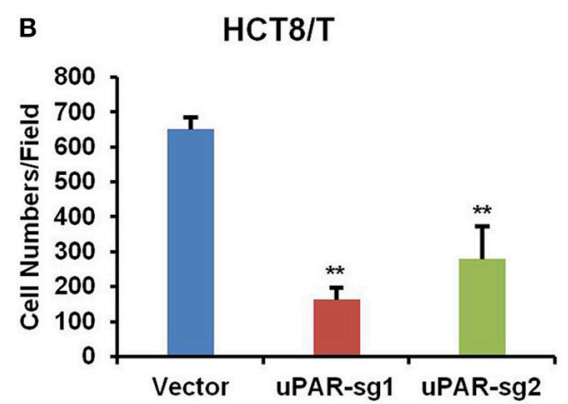

D

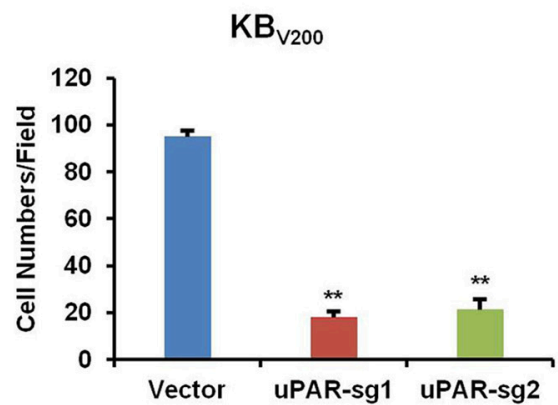

FIGURE 5 | Knockout of UPAR inhibits cell invasion. Cell invasion was determined with transwell assay. Representative invasion images and quantification of HCT8/T $(\mathbf{A}, \mathbf{B})$ and $K_{B_{2} 00}(\mathbf{C}, \mathbf{D})$ cells were shown. ${ }^{* *} P<0.01$ vs. corresponding control.
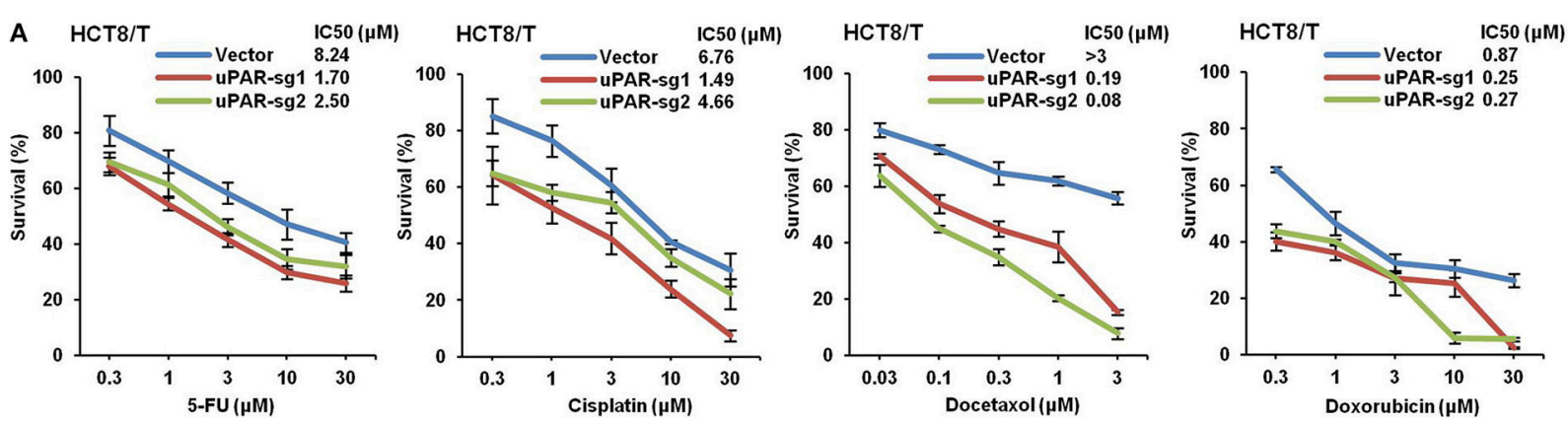

B

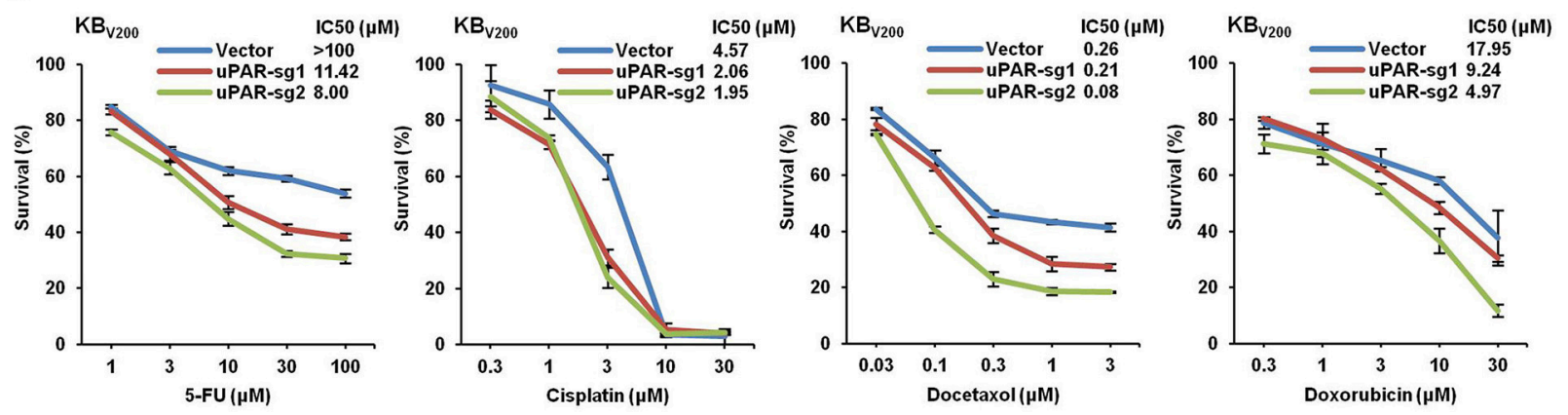

FIGURE 6 | Knockout of UPAR decreases multidrug resistance. Cells survival was measured by MTT assay. The representative growth curve of HCT8/T (A) and $\mathrm{KB}_{\mathrm{V} 200}$ (B) cells treated with the indicated concentrations of 5-FU, cisplatin, docetaxel, and doxorubicin for $72 \mathrm{~h}$ were shown. 
CRISPR/Cas9 system has been widely applied in exploring the molecular mechanism of tumorigenesis, generating the models for cancer research and identifying the targets for cancer treatment, etc. A genome-wide CRISPR screen shows that lossof-function mutations of some genes including NF2, PTEN, CDKN2A, TRIM72, FGA, miR-152, miR-345, and so on are able to drive tumor growth and metastasis in a mouse model (24). Using CRISPR/Cas9 technology to target MAN2A1-FER fusion gene inhibits tumor proliferation and metastasis in the mouse models of prostate and liver cancer (25). Colorectal cancer from normal human intestinal epithelium organoids are generated by introducing mutations in the tumor suppressor genes APC, SMAD4 and TP53, and oncogenes KRAS and/or PIK3CA with CRISPR/Cas9 system $(26,27)$. Liver tumors in mice are occurred by using hydrodynamic injection of CRISPR/Cas9 plasmids and sgRNAs that directly target the tumor suppressor genes PTEN and p53 (28). Mouse pancreatic ductal adenocarcinoma models are established by introducing 13 sgRNAs of different tumor suppressor genes into expression vectors and then transferred them to mouse pancreatic tissue (29). CDC25A is identifies as a determinant of sensitivity to ATR inhibitors by a genomewide CRISPR screen (30). Deletion of genes such as NF1 and MED12 with CRISPR/Cas9 system is associated with resistance to vemurafenib (31). Moreover, the combination of CRISPR/Cas9 gene editing technology and immunotherapy, especially with CAR-T cell therapy, will have enormous therapeutic potential in leukemia, lymphoma, and some solid tumors $(32,33)$. Using CRISPR/Cas9 system can produce universal CAR-T cells by simultaneously targeting TCR and HLA-I (34) and enhanced CAR-T cells by deleting $\mathrm{T}$ cell inhibitory receptor or signaling molecule genes such as PD1 and CTLA4 $(33,35)$. We previously have demonstrated that targeting $\mathrm{ABCB} 1$ by CRISPR/Cas9-based

\section{REFERENCES}

1. Mahmood N, Mihalcioiu C, Rabbani SA. Multifaceted role of the urokinase-type plasminogen activator (uPA) and its receptor (uPAR): diagnostic, prognostic, and therapeutic applications. Front Oncol. (2018) 8:24. doi: 10.3389/fonc.2018.00024

2. Smith HW, Marshall CJ. Regulation of cell signalling by uPAR. Nat Rev Mol Cell Biol. (2010) 11:23-36. doi: 10.1038/nrm2821

3. Noh H, Hong S, Huang S. Role of urokinase receptor in tumor progression and development. Theranostics. (2013) 3:487-95. doi: 10.7150/thno.4218

4. Setyono-Han B, Sturzebecher J, Schmalix WA, Muehlenweg B, Sieuwerts AM, Timmermans M, et al. Suppression of rat breast cancer metastasis and reduction of primary tumour growth by the small synthetic urokinase inhibitor WX-UK1. Thromb Haemost. (2005) 93:779-86. doi: 10.1160/TH04-11-0712

5. Zhu M, Gokhale VM, Szabo L, Munoz RM, Baek H, Bashyam S, et al. Identification of a novel inhibitor of urokinase-type plasminogen activator. Mol Cancer Ther. (2007) 6:1348-56. doi: 10.1158/1535-7163.MCT-06-0520

6. Heinemann V, Ebert MP, Pinter T, Bevan P, Neville NG, Mala C. Randomized phase II trial with an uPA inhibitor (WX-671) in patients with locally advanced nonmetastatic pancreatic cancer. J Clin Oncol. (2010) 28:4060. doi: 10.1200/jco.2010.28.15_suppl.4060

7. Andersen LM, Wind T, Hansen HD, Andreasen PA. A cyclic peptidylic inhibitor of murine urokinase-type plasminogen activator: changing species specificity by substitution of a single residue. Biochem J. (2008) 412:447-57. doi: 10.1042/BJ20071646 genome editing reverses $\mathrm{ABCB} 1$-mediated multidrug resistance in cancer cells, resulting in the increase of the sensitivity and intracellular accumulation of the anti-cancer drugs (36). Although there are several limitations such as off-targets and delivery in the clinical application of CRISPR/Cas9 technology, it is believed that CRISPR/Cas9 system will benefit cancer patients in the near future.

In summary, our results have demonstrated that targeting uPAR by CRISPR/Cas9-based genome editing causes knockout of UPAR in human cancer cell lines, resulting in attenuation of cell proliferation, migration, invasion and multidrug resistance. Our study offers valuable evidences for the role of uPAR in cancer malignancy and drug resistance.

\section{AUTHOR CONTRIBUTIONS}

$\mathrm{KW}, \mathrm{Z}-\mathrm{HX}$, and ZS designed the experiments, performed the experiments, analyzed the data, and wrote the paper. Q-WJ, YY, J-RH, M-LY, M-NW, YL, S-TW, and KL performed the experiments. All authors read and approved the final manuscript.

\section{FUNDING}

This work was supported by funds from the National Key Research and Development Program of China No. 2017YFA0505104 ZS, the National Natural Science Foundation of China No. 81772540 ZS, the Guangdong Natural Science Funds for Distinguished Young Scholar No. 2014A030306001 ZS, the Guangdong Special Support Program for Young Talent No. 2015TQ01R350 ZS, the Science and Technology Program of Guangdong No. 2016A050502027 ZS, the Science and Technology Program of Guangzhou No. 201704030058 ZS.

8. Ploug M, Ostergaard S, Gardsvoll H, Kovalski K, Holst-Hansen C, Holm A, et al. Peptide-derived antagonists of the urokinase receptor. Affinity maturation by combinatorial chemistry, identification of functional epitopes, and inhibitory effect on cancer cell intravasation. Biochemistry. (2001) 40:12157-68. doi: 10.1021/bi010662g

9. Luparello C, Del Rosso M. In vitro anti-proliferative and anti-invasive role of aminoterminal fragment of urokinase-type plasminogen activator on 8701-BC breast cancer cells. Eur J Cancer. (1996) 32A:702-7. doi: 10.1016/0959-8049(95)00657-5

10. Mazar AP, Ahn RW, O'Halloran TV. Development of novel therapeutics targeting the urokinase plasminogen activator receptor (uPAR) and their translation toward the clinic. Curr Pharm Des. (2011) 17:1970-8. doi: 10.2174/138161211796718152

11. Ahmed N, Oliva K, Wang Y, Quinn M, Rice G. Downregulation of urokinase plasminogen activator receptor expression inhibits Erk signalling with concomitant suppression of invasiveness due to loss of uPAR-beta 1 integrin complex in colon cancer cells. Brit J Cancer. (2003) 89:374-84. doi: $10.1038 /$ sj.bjc. 6601098

12. Zhang F, Tom CC, Kugler MC, Ching TT, Kreidberg JA, Wei Y, et al. Distinct ligand binding sites in integrin alpha 3 beta 1 regulate matrix adhesion and cell-cell contact. J Cell Biol. (2003) 163:177-88. doi: 10.1083/jcb.200 304065

13. Ghosh S, Johnson JJ, Sen R, Mukhopadhyay S, Liu Y, Zhang F, et al. Functional relevance of urinary-type plasminogen activator receptoralpha3betal integrin association in proteinase regulatory pathways. $J$ Biol Chem. (2006) 281:13021-9. doi: 10.1074/jbc.M508526200 
14. Kenny HA, Leonhardt P, Ladanyi A, Yamada SD, Montag A, Im $\mathrm{HK}$, et al. Targeting the urokinase plasminogen activator receptor inhibits ovarian cancer metastasis. Clin Cancer Res. (2011) 17:459-71. doi: 10.1158/1078-0432.CCR-10-2258

15. Barrangou R, Doudna JA. Applications of CRISPR technologies in research and beyond. Nat Biotechnol. (2016) 34:933-41. doi: 10.1038/nbt.3659

16. Fellmann C, Gowen BG, Lin PC, Doudna JA, Corn JE. Cornerstones of CRISPR-Cas in drug discovery and therapy. Nat Rev Drug Discov. (2017) 16:89-100. doi: 10.1038/nrd.2016.238

17. Yuan ML, Li P, Xing ZH, Di JM, Liu H, Yang AK, et al. Inhibition of WEE1 suppresses the tumor growth in laryngeal squamous cell carcinoma. Front Pharmacol. (2018) 9:1041. doi: 10.3389/fphar.2018.01041

18. Rysenkova KD, Semina EV, Karagyaur MN, Shmakova AA, Dyikanov DT, Vasiluev PA, et al. CRISPR/Cas9 nickase mediated targeting of urokinase receptor gene inhibits neuroblastoma cell proliferation. Oncotarget. (2018) 9:29414-30. doi: 10.18632/oncotarget.25647

19. Gutova M, Najbauer J, Gevorgyan A, Metz MZ, Weng Y, Shih CC, et al. Identification of uPAR-positive chemoresistant cells in small cell lung cancer. PLoS ONE. (2007) 2:e243. doi: 10.1371/journal.pone.0000243

20. Cortes-Dericks L, Carboni GL, Schmid RA, Karoubi G. Putative cancer stem cells in malignant pleural mesothelioma show resistance to cisplatin and pemetrexed. Int J Oncol. (2010) 37:437-44. doi: 10.3892/ijo-0000692

21. Huang Z, Wang L, Wang Y, Zhuo Y, Li H, Chen J, et al. Overexpression of CD147 contributes to the chemoresistance of head and neck squamous cell carcinoma cells. J Oral Pathol Med. (2013) 42:541-6. doi: 10.1111/jop.12046

22. Eastman BM, Jo M, Webb DL, Takimoto S, Gonias SL. A transformation in the mechanism by which the urokinase receptor signals provides a selection advantage for estrogen receptor-expressing breast cancer cells in the absence of estrogen. Cell Signal. (2012) 24:1847-55. doi: 10.1016/j.cellsig.201 2.05 .011

23. Zhou J, Kwak KJ, Wu Z, Yang D, Li J, Chang M, et al. PLAUR confers resistance to gefitinib through EGFR/P-AKT/Survivin signaling pathway. Cell Physiol Biochem. (2018) 47:1909-24. doi: 10.1159/000491071

24. Chen S, Sanjana NE, Zheng K, Shalem O, Lee K, Shi X, et al. Genome-wide CRISPR screen in a mouse model of tumor growth and metastasis. Cell. (2015) 160:1246-60. doi: 10.1016/j.cell.2015.02.038

25. Chen ZH, Yu YP, Zuo ZH, Nelson JB, Michalopoulos GK, Monga S, et al. Targeting genomic rearrangements in tumor cells through Cas9mediated insertion of a suicide gene. Nat Biotechnol. (2017) 35:543-50. doi: $10.1038 /$ nbt.3843

26. Matano M, Date S, Shimokawa M, Takano A, Fujii M, Ohta Y, et al. Modeling colorectal cancer using CRISPR-Cas9-mediated engineering of human intestinal organoids. Nat Med. (2015) 21:256-62. doi: 10.1038/nm.3802
27. Drost J, van Jaarsveld RH, Ponsioen B, Zimberlin C, van Boxtel R, Buijs A, et al. Sequential cancer mutations in cultured human intestinal stem cells. Nature. (2015) 521:43-7. doi: 10.1038/nature14415

28. Xue W, Chen S, Yin H, Tammela T, Papagiannakopoulos T, Joshi NS, et al. CRISPR-mediated direct mutation of cancer genes in the mouse liver. Nature. (2014) 514:380-4. doi: 10.1038/nature13589

29. Maresch R, Mueller S, Veltkamp C, Oellinger R, Friedrich M, Heid I, et al. Multiplexed pancreatic genome engineering and cancer induction by transfection-based CRISPR/Cas9 delivery in mice. Nat Commun. (2016) 7:10770. doi: 10.1038/ncomms 10770

30. Ruiz S, Mayor-Ruiz C, Lafarga V, Murga M, Vega-Sendino M, Ortega $\mathrm{S}$, et al. A genome-wide CRISPR screen identifies CDC25A as a determinant of sensitivity to ATR inhibitors. Mol Cell. (2016) 62:307-13. doi: 10.1016/j.molcel.2016.03.006

31. Shalem O, Sanjana NE, Hartenian E, Shi X, Scott DA, Mikkelsen TS, et al. Genome-Scale CRISPR-Cas9 knockout screening in human cells. Science. (2014) 343:84-7. doi: 10.1126/science.1247005

32. Maus MV, Grupp SA, Porter DL, June CH. Antibody-modified T cells: CARs take the front seat for hematologic malignancies. Blood. (2014) 123:2625-35. doi: 10.1182/blood-2013-11-492231

33. Ren JT, Zhao YB. Advancing chimeric antigen receptor T cell therapy with CRISPR/Cas9. Protein Cell. (2017) 8:634-43. doi: 10.1007/s13238-017-0410-x

34. Ren JT, Liu XJ, Fang CY, Jiang SG, June CH, Zhao YB. Multiplex genome editing to generate universal CAR T cells resistant to PD1 inhibition. Clin Cancer Res. (2017) 23:2255-66. doi: 10.1158/1078-0432.CCR-16-1300

35. Hoos A. Development of immuno-oncology drugs - from CTLA4 to PD1 to the next generations. Nat Rev Drug Discov. (2016) 15:235-47. doi: 10.1038/nrd.2015.35

36. Yang Y, Qiu JG, Li Y, Di JM, Zhang WJ, Jiang QW, et al. Targeting ABCB1mediated tumor multidrug resistance by CRISPR/Cas9-based genome editing. Am J Transl Res. (2016) 8:3986-94.

Conflict of Interest Statement: The authors declare that the research was conducted in the absence of any commercial or financial relationships that could be construed as a potential conflict of interest.

Copyright (c) 2019 Wang, Xing, Jiang, Yang, Huang, Yuan, Wei, Li, Wang, Liu and Shi. This is an open-access article distributed under the terms of the Creative Commons Attribution License (CC BY). The use, distribution or reproduction in other forums is permitted, provided the original author(s) and the copyright owner(s) are credited and that the original publication in this journal is cited, in accordance with accepted academic practice. No use, distribution or reproduction is permitted which does not comply with these terms. 